\title{
Underwater Cloth Simulation with Fractional Derivatives
}

\author{
OKTAR OZGEN, MARCELO KALLMANN, LYNNETTE ES RAMIREZ and CARLOS FM COIMBRA \\ University of California, Merced
}

\begin{abstract}
We introduce the use of fractional differentiation for simulating cloth deformations underwater. The proposed approach is able to achieve realistic underwater deformations without simulating the Eulerian body of water in which the cloth is immersed. Instead, we propose a particle-based cloth model where half-derivative viscoelastic elements are included for describing both the internal and external dynamics of the cloth. These elements model the cloth responses to fluid stresses and are also able to emulate the memory-laden behavior of particles in a viscous fluid. As a result, we obtain fractional clothes, which are able to correctly depict the dynamics of the immersed cloth interacting with the fluid even though the fluid is not simulated. The proposed approach produces realistic underwater cloth deformations and has obvious advantages in simplicity and speed of computation in comparison to volumetric fluid simulation approaches.
\end{abstract}

Categories and Subject Descriptors: I.3.7 [Computer Graphics]: ThreeDimensional Graphics and Realism-Animation; I.3.5 [Computer Graphics]: Computational Geometry and Object Modeling-Physically based modeling

Additional Key Words and Phrases: cloth simulation, physically based animation, underwater simulation, fractional derivatives.

\section{INTRODUCTION}

Cloth simulation has been a central topic in physically-based computer animation. Since the introduction of the first models of deformable surfaces [Terzopoulos et al. 1987; Terzopoulos and Fleischer 1988] several research groups have achieved impressive cloth simulation results and their effects are now commonly seen in computer-generated movies and even in interactive applications such as in 3D computer games. Although several extensions have been proposed in the cloth simulation domain, no specific attention has been given for extending the regular spring mesh cloth model for producing deformations that simulate underwater behavior.

University of California, Merced

5200 N. Lake Road - Merced CA 95343.

e-mails: \{oozgen, mkallmann, lramirez, ccoimbra\}@ucmerced.edu

Permission to make digital or hard copies of part or all of this work for personal or classroom use is granted without fee provided that copies are not made or distributed for profit or commercial advantage and that copies show this notice on the first page or initial screen of a display along with the full citation. Copyrights for components of this work owned by others than ACM must be honored. Abstracting with credit is permitted. To copy otherwise, to republish, to post on servers, to redistribute to lists, or to use any component of this work in other works requires prior specific permission and/or a fee. Permissions may be requested from Publications Dept., ACM, Inc., 2 Penn Plaza, Suite 701, New York, NY 10121-0701 USA, fax +1 (212) 869-0481, or permissions@ @acm.org.

(c) YYYY ACM 0730-0301/YYYY/10-ARTXXX \$10.00

DOI 10.1145/XXXXXXX.YYYYYYY

http://doi.acm.org/10.1145/XXXXXXX.YYYYYYY
One logical approach for simulating cloth underwater is to embed the cloth model in a fluid simulation system. Such a coupled system can then be solved in different ways, for instance by coupling the Eulerian fluid to a Lagrangian (particle-based) cloth [RobinsonMosher et al. 2008], or also by a fully Lagrangian model [Müller et al. 2004]. Although required for accurately modeling the problem, such approaches are expensive to compute as they perform volumetric simulations of the Eulerian fluid surrounding the cloth surface. In particular for the cases when only the deformations of the immersed cloth are needed, a simplified simulation model would be much more practical.

This paper builds on the key observation that the total force acting on an oscillating particle immersed in a Newtonian fluid is composed of force contributions which are proportional to: 1) the acceleration of the displaced fluid (the virtual mass force), 2) the velocity of the particle (the Stokes drag), and 3) the so-called history (or Basset) force, which accounts for history effects of the moving flow around the particle. These contributions can be derived exactly from the Navier-Stokes equations for the limit of small particles in viscous fluids at low Reynolds numbers. Most importantly, Coimbra and Rangel [1998] have showed that the Basset force is mathematically equivalent to the half-derivative of the differential velocity between the particle and the far-stream flow.

Motivated by these observations we propose a cloth deformation model which employs the concept of non-integer derivatives for achieving underwater deformation effects. By doing so we obtain fractional clothes, which are able to simulate the dynamics of a network of immersed particles without performing expensive volumetric fluid simulations. The complex inertial-viscous balance between the cloth and the fluid is computed in our model by half-derivative terms, which dynamically modulate the cloth damping according to the previous states of the cloth, i. e., the cloth history.

After discussing related work in the following section we provide in Section 3 an overview of Fractional Calculus for modeling the motion of particles. Our fractional cloth model is then presented in Section 4. Finally, Section 5 presents and discusses the several obtained results and Section 6 concludes the paper.

\section{RELATED WORK}

Cloth simulation is a main topic in computer animation and a rich literature is available [Carignan et al. 1992; Breen et al. 1992; Provot 1995; Eberhardt et al. 1996; Volino et al. 1995; Baraff and Witkin 1998; House and Breen 2000; Choi and Ko 2002; Terzopoulos et al. 1987; Terzopoulos and Fleischer 1988]. We focus our review on how damping has been used in previous works.

Damping has always been an important aspect of cloth simulation systems. It has been mainly addressed as a viscous drag force with the goal of resisting motion, making particles gradually come to rest in the absence of other influences [Baraff and Witkin 1997]. The viscous drag force is generally perceived as an external force proportional to the velocity: $\boldsymbol{F}=-k_{d} \dot{\boldsymbol{x}}$ [Terzopoulos et al. 1987; 

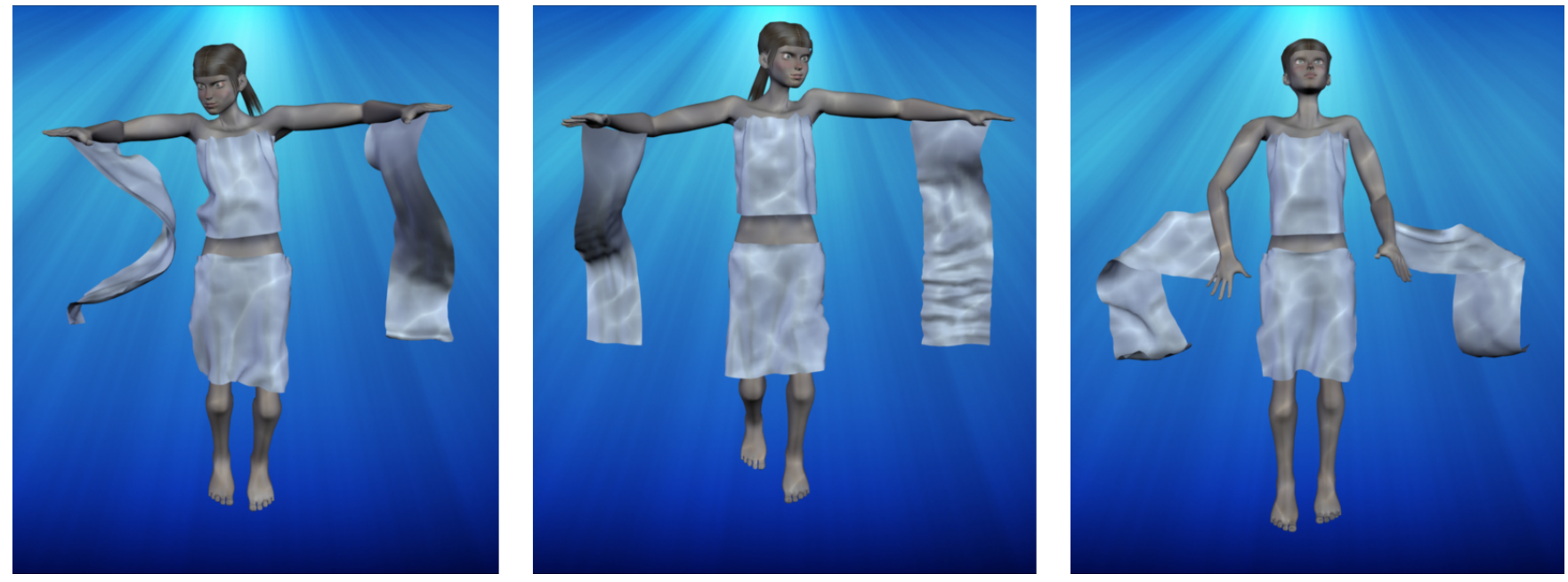

Fig. 1. Our cloth model based on fractional derivatives is able to achieve realistic underwater deformation behavior.

Terzopoulos and Fleischer 1988; Provot 1995], and is sometimes mentioned to be applied opposing the normal direction of the cloth surface [Baraff and Witkin 1998; Selle et al. 2009].

Damping has also been used as an internal force applied to particles from interaction with other particles. This type of damping is usually coupled with stretch, shear and bend forces, and is justified by the several benefits to the simulation: numerical stability, oscillation prevention [Baraff and Witkin 1998] and intrinsic damping property of fabrics [Choi and Ko 2002]. The damping force in this case is proportional to the relative velocity between a pair of connected particles, and is usually computed as $\boldsymbol{F}_{i j}=-k_{d}\left(\dot{\boldsymbol{x}}_{i}-\dot{\boldsymbol{x}}_{j}\right)$, where $\dot{\boldsymbol{x}}_{i}$ and $\dot{\boldsymbol{x}}_{j}$ are the velocities of the interacting particles $i$ and $j$ [Desbrun et al. 1999; Choi and Ko 2002; Hauth et al. 2003].

One particular way of including internal damping forces is proposed by Baraff and Witking [1998], where the damping forces only depend on the components of the velocities in the directions of each maintained condition (stretch, shear and bend). In contrast, other works are less detailed in the description of the used damping mechanism [Eberhardt et al. 1996; Volino et al. 1995].

Models have also been proposed for achieving realistic cloth deformations under air flows. In particular, Ling et al. [1996] and Ling [2000] consider incompressible, irrotational and inviscid airflows, and employ two aerodynamic models to compute forces in response to the airflows. They used a quasi-steady force model to deduce the airflow effects from constant or slowly changing free stream velocities. An unsteady force model is used for the cases where the free stream velocity of the air flow changes rapidly and the flow relative to the cloth becomes unsteady.

Tu and Terzopoulos [1994] describe one of the few works attempting to achieve underwater behaviors with a spring-mass system. They incorporate external forces which depend on the relative velocity between the surface of a deformable fish and the fluid. These forces are normal to the fish surface (similarly to a damping force) and are used to achieve realistic locomotion underwater. The new half-derivative terms introduced by our work include additional fluid responses and could also be used to achieve locomotion patterns.
Specific Contributions We propose in this paper the concept of employing damping forces which are modulated by half-derivative viscoelastic elements that simulate the Basset drag in Stokes flows. As explained in Section 3, our viscoelastic elements are made proportional to the half-derivative of the displacements in order to capture the macroscopic behavior of the whole cloth underwater. Our approach elegantly models the most important contributions of the history drag on the motion of the cloth and is able to produce realistic underwater deformations simulating the viscoelastic cloth-fluid interaction. We also address for the first time the topic of simulating underwater cloth behavior without recurring to a volumetric fluid simulation system.

\section{FRACTIONAL CALCULUS AND PARTICLE MOTION}

The subject of Fractional Calculus (FC) [Oldham and Spanier 1974], or the mathematical analysis of differentiation and integration to an arbitrary non-integer order, has attracted much interest in the past three decades. The traditional name of the discipline is a misnomer since any non-integer (rational or irrational) order of differentiation and integration is easily accounted for within the context of generalized differentiation.

Physical evidence of fractional order behavior has been demonstrated in diverse areas, which include fluid and solid mechanics, rheology, electromagnetism, electrochemistry, and biology to mention a few. Beyond the fact that FC models provide superior modeling capability of memory-intense and delay systems, fractional modeling has been associated with the exact description of unsteady viscous and viscoelastic phenomena. Of particular importance to our work is the ability of non-integer derivatives to capture non-local behavior, and to interpolate between distinct dynamic regimes such as between the elastic and the viscous behavior [Oldham and Spanier 1974; Miller and Ross 1993; Podlubny 1999; Hu 2005; Hilfer 2000; Kilbas et al. 2006].

For instance, Coimbra et al. [2004] and L'Esperance et al. [2005] provide definitive experimental evidence of fractional history effects in the unsteady viscous motion of small particles in suspension [Coimbra C. F. M. and Rangel R. H. 1998; Coimbra C. F. M. and Kobayashi M. H. 2002]. It is this same behavior [Coimbra C. 

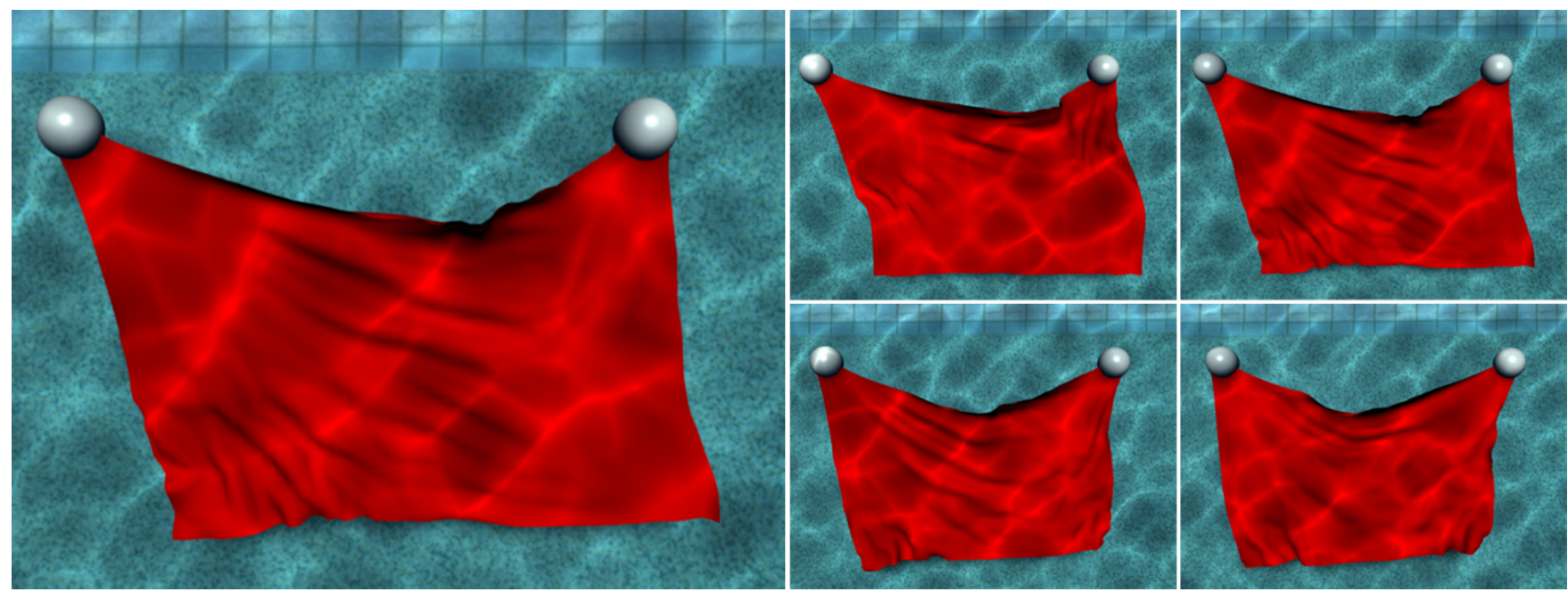

Fig. 2. Examples of internal effects produced by the viscoelastic spring elements in our fractional cloth formulation.

F. M. et al. 2004] that we propose to model by adding a fractional term to the equation of motion of particles in a cloth mesh moving underwater. The goal is to simulate the history-laden dynamics of the deformable cloth by introducing a non-local dynamic operator that accounts for an intermediate response between position and velocity of the medium. This viscoelastic term of order $1 / 2$ is able to simulate the fluid response to cloth accelerations without the complication of simulating the entire hydroelastic process.

The motivation for our approach to simulate cloth-fluid interactions follows from the study of individual particles moving in a viscous fluid [Coimbra C. F. M. and Rangel R. H. 1998]. Stokes [1845] pioneered the study of viscous particle motion by determining the force acting on a small fixed particle that is subjected to a uniform fluid velocity. Stokes related the undisturbed flow (or far-stream conditions) to the force acting on the sphere, thus creating a direct relationship between the resulting force (or torque) acting on a spherical particle and the kinematics of the free-stream flow.

Mathematically, Stokes determined the operator $\Lambda_{S}$ that relates the force $\boldsymbol{F}$ acting on a particle to the background flow field $\boldsymbol{U}$, such that an expression of the form $\boldsymbol{F}=\Lambda_{S}(\boldsymbol{U})$ is determined. This is a major accomplishment because it allows for the calculation of the forces acting on the particle by considering only the undisturbed flow conditions as opposed to relating the force to the spatially dependent, non-uniform flow in the vicinity of the particle. The computation simplification of Stokes' analysis is such that, under special circumstances, one can predict accurately the rate of sedimentation of small particles without ever resolving or understanding the flow generated by the motion of the particles.

The resulting Stokes drag formula relates the force exerted on the sphere to the constant free-stream velocity $\boldsymbol{U}$, the dynamical viscosity of the fluid $\mu$, and the radius $a$ of the sphere in a linear way, given that the particle Reynolds number is maintained much smaller than unity.

Boussinesq [1885] and Basset [1888] independently extended Stokes' derivation to a case where the particle accelerates through the fluid due to a constant gravitational force but still neglecting the convective terms in the Navier-Stokes equation (valid only for small Reynolds numbers). The particle equation of motion with a constant forcing (the gravity term) is sometimes referred to as the $\mathrm{BBO}$ equation, due to the original contributions of Boussinesq, Basset and Oseen.

The BBO equation is an integro-differential equation that has a removable singularity in the integrand of the history term. The history or fractional term can be then derived directly from the Stokes operator $\Lambda_{S}$ using Duhamel's Superposition Theorem. The history term is found to be simply $a \Lambda_{S} \nu^{-1 / 2} D^{1 / 2} \boldsymbol{V}$, where $\nu$ is the kinematic viscosity of the fluid and $D^{1 / 2} \boldsymbol{V}$ represents the half-derivative of the particle velocity [Coimbra C. F. M. and Rangel R. H. 1998], which can be computed with the following Riemann-Liouville differential operator:

$$
D^{1 / 2} \boldsymbol{V}=\frac{1}{\Gamma(1 / 2)} \int_{-\infty}^{t}(t-\sigma)^{-1 / 2} \frac{d \boldsymbol{V}(\boldsymbol{\sigma})}{d t} d \sigma,
$$

where $\Gamma$ is the generalized factorial function and $\Gamma(1 / 2)=\sqrt{\pi}$.

The results above are important because they allow us to replace the complicated, evolving flow field around the particle with an integral force that can be calculated in an iterative way. The implications of this formulation in terms of computational time are clear. The fractional force plays the role of an intermediate dynamic variable between velocity and acceleration, and is exact for a single particle in low Reynolds flows.

We are however interested in the overall effect of a piece of cloth interacting with the flow. One key observation is that the immersed cloth will have its internal structures mixed with molecules of water and therefore both its constitutive material and its response with the surrounding volume of water will be better described as a flowable microstructure similar to the ones studied in Rheology [Larson 1998]. Our immersed cloth will therefore be better described as a viscoelastic microstructure exhibiting obvious fluid-like (viscous) and also solid-like (elastic) behaviors.

While an exact model for the immersed cloth microstructure cannot be devised, we recall that the intermediate behavior between viscosity and elasticity is, in its simplest form, a viscoelastic effect that can be approximated by a term on the half-derivative of the displacement [Coimbra C. F. M. 2003]. We therefore translate the particle history effect described by the half-derivative in Equation 1 

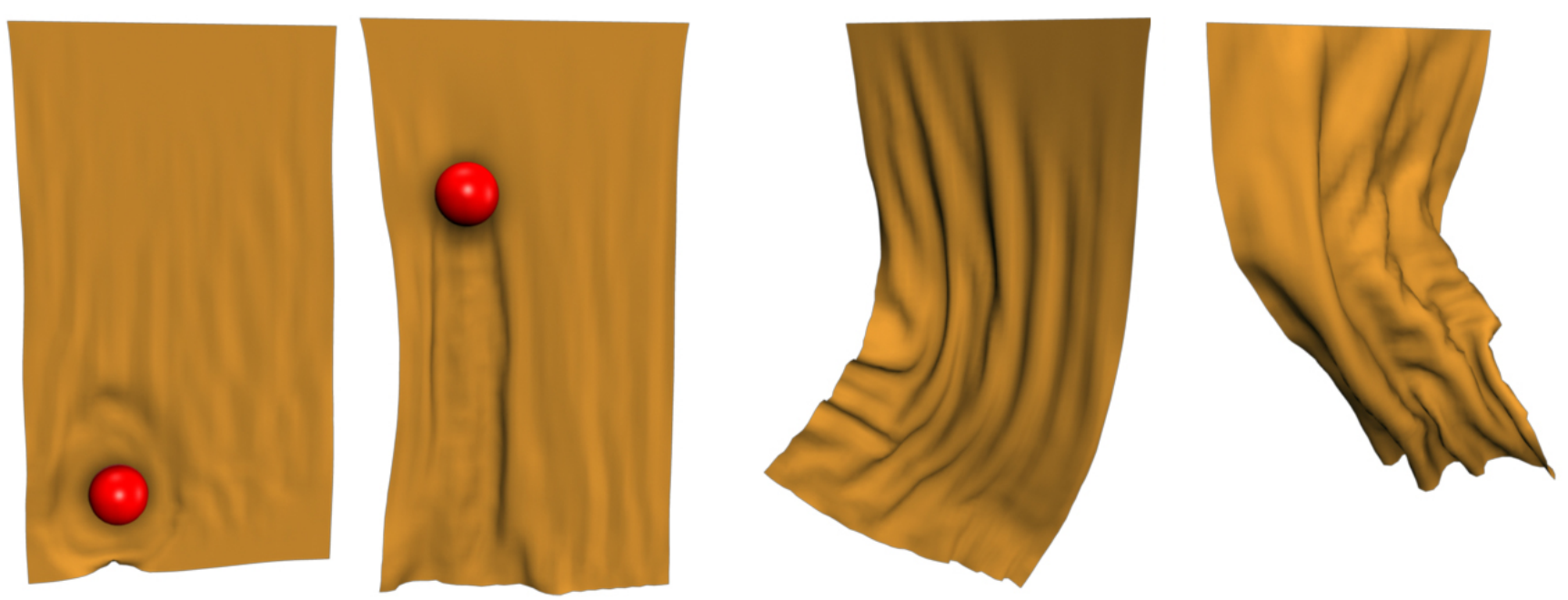

Fig. 3. The left two figures show consistent propagation of waves caused by a sphere interacting with the cloth model. The right two figures show examples obtained with the cloth top edge being energetically moved sideways. The obtained long lasting wave propagations demonstrate consistent cloth-water inertial responses captured by the viscoelastic half-derivative terms of our fractional cloth model.

into the immersed cloth by applying the half-derivative operator to the mesh displacements (instead of applying to the relative velocity).

The result is a viscoelastic term which is added to both the internal forces and the external drag of the cloth. The internal forces then become governed by viscoelastic spring elements and the external drag becomes the history (or Basset) drag. As a result, the achieved model simulates the different inertial responses between the fluid and the cloth in an efficient way. The following section describes our model in detail.

\section{FRACTIONAL CLOTHES}

The mesh structure of our cloth model is based on the scheme presented by [Choi and Ko 2002]. The cloth is represented as a quadrilateral mesh of particles. Instead of regular damped springs, our particles are connected to each other by massless viscoelastic spring elements. There are three types of spring elements, which are responsible for the stretch, shear and bend forces. The connectivity of the springs is described in the following way: a particle indexed by $p(i, j)$ is connected by stretch springs to its neighbor particles indexed by $p(i \pm 1, j), p(i, j \pm 1)$. The shear springs connect the particle to $p(i \pm 1, j \pm 1)$, and the bend springs connect the particle to $p(i \pm 2, j), p(i, j \pm 2)$ and $p(i \pm 2, j \pm 2)$.

\subsection{Forces}

The dynamics of the system is governed by Newton's second law of motion $\boldsymbol{F}=m \boldsymbol{a}$, where $m$ is the mass and $\boldsymbol{a}$ is the acceleration of a particle. The acceleration is computed at every time step based on the total force $\boldsymbol{F}$ applied to the particle, and which accounts for all external and internal forces.

The included external forces are: the gravitational force, the buoyancy of water, a regular viscous drag, and the history drag. The gravitational force and the buoyancy are computed together with:

$$
\boldsymbol{F}_{g}=\left(\rho_{c}-\rho_{w}\right) V_{c} \boldsymbol{g},
$$

where $\rho_{c}$ is the density of a cloth particle, $\rho_{w}$ is the water density (approximately $1000 \mathrm{~kg} / \mathrm{m}^{3}$ ), $V_{c}$ is an estimation of the volume of one cloth particle and $\boldsymbol{g}$ is the gravitational acceleration. The specification of different material densities will lead to different behaviors, ranging from a material which floats to one which quickly sinks. Correctly specifying the density of a wet material is not trivial; however, for the scope of computer animation applications the density can be seen as a parameter which can be fine-tuned in order to achieve different material properties.

External forces for modeling underwater currents can also be added to the global $\boldsymbol{F}_{g}$ force term. In our simulations, we tuned $\rho_{c}$ and $V_{c}$ such that the particles slightly sink in direction of the gravity force, similar to the behavior observed in real submerged clothes (see Figure 5). $\boldsymbol{F}_{g}$ is considered to be constant in the scope of this paper.

The regular viscous drag is responsible for dissipating kinetic energy and is modeled with:

$$
\boldsymbol{F}_{v d}=-k_{v d} \dot{\boldsymbol{x}}
$$

where $k_{v d}$ is the viscous drag coefficient and $\dot{\boldsymbol{x}}=\boldsymbol{v}$, is the velocity of the particle. Here we employ the viscous drag force opposing the velocity vector since this choice has showed to produce better results than other options, as for instance using a force opposing the surface normal. The history drag is then added to account for the inertial responses with the fluid and is computed as:

$$
\boldsymbol{F}_{h d}=-k_{h d}\left(D^{1 / 2} \boldsymbol{x} \cdot \hat{\boldsymbol{v}}\right) \hat{\boldsymbol{v}}
$$

where $k_{h d}$ is the history drag coefficient and $\hat{\boldsymbol{v}}=\boldsymbol{v} /|\boldsymbol{v}|$ is the unit velocity vector.

As discussed in Section 3, the history drag is a half-derivative of the displacements, however note that the drag force should oppose the direction of movement and therefore Equation 4 projects the computed half-derivative in the velocity direction. By including the viscous drag and the history drag with different coefficients a greater flexibility can be obtained in fine-tuning the overall drag effect. 

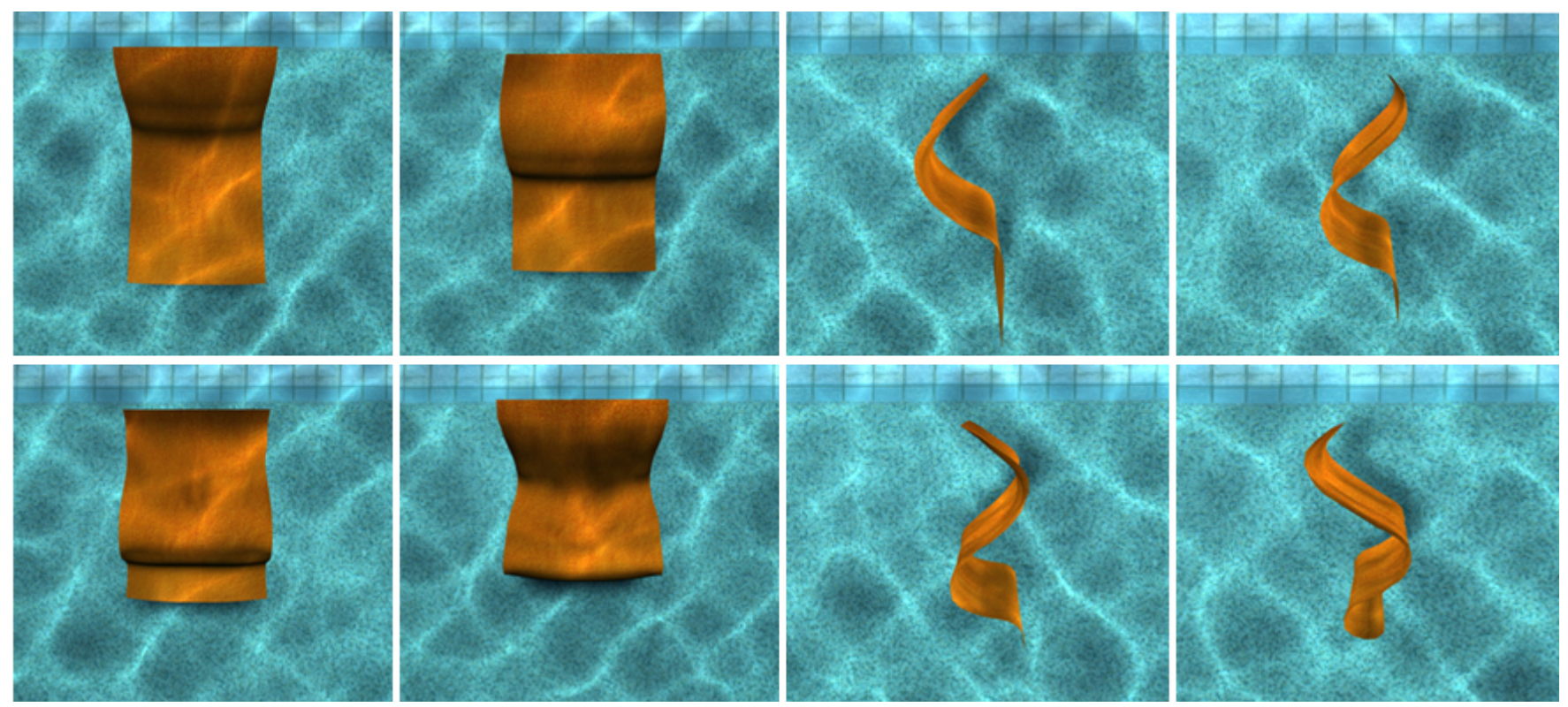

Fig. 4. The cyclic motion applied to the top of this simulated piece of cloth generates realistic bump propagations.

The internal forces acting on a particle are the forces which originate from the stretch, bend and shear viscoelastic spring elements connected to each particle. The spring forces are responsible for all the internal deformation responses of the cloth and their formulation also includes a half-derivative term. The spring force $\boldsymbol{F}_{s}$ exerted on particle $i$ by the fractional viscoelastic spring element that links particle $i$ to particle $j$, is formulated as:

$$
\boldsymbol{F}_{s}=-k_{s}(|\boldsymbol{l}|-r) \frac{\boldsymbol{l}}{|\boldsymbol{l}|}-k_{d}\left[\frac{\left(D^{1 / 2} \boldsymbol{x}_{i}-D^{1 / 2} \boldsymbol{x}_{j}\right) \cdot \boldsymbol{l}}{|\boldsymbol{l}|}\right] \frac{\boldsymbol{l}}{|\boldsymbol{l}|}
$$

where $\boldsymbol{l}=\boldsymbol{x}_{\boldsymbol{i}}-\boldsymbol{x}_{\boldsymbol{j}}$ is the difference between the positions of the two particles, $r$ is the rest length of the spring, $k_{d}$ is the viscoelastic damping constant and $k_{s}$ is the linear spring constant. Note that while a regular damped spring would have the damping proportional to the first time derivative of the position, our spring element has the damping proportional to the half-derivatives $D^{1 / 2} \boldsymbol{x}_{i}$ and $D^{1 / 2} \boldsymbol{x}_{j}$, achieving the memory-laden viscoelastic damping term.

\subsection{Computing the Half Derivative Terms}

Although the history integral of Equation 1 is singular, it can be numerically integrated with a consistent, first-order accurate, approximation suggested by Coimbra [2003], which is an adaptation of the algorithm for general convolution of integrals. The $1 / 2$ order derivative of $\boldsymbol{x}$ can thus be expressed as:

$$
\begin{aligned}
D^{1 / 2} \boldsymbol{x}_{n} & =\frac{h}{6 \sqrt{\pi}} \sum_{i=1}^{n-1}\left[\frac{\dot{\boldsymbol{x}}_{i-1}}{(n h-(i-1) h)^{1 / 2}}\right. \\
& \left.+\frac{2\left(\dot{\boldsymbol{x}}_{i-1}+\dot{\boldsymbol{x}}_{i}\right)}{(n h-(i-1 / 2) h)^{1 / 2}}+\frac{\dot{\boldsymbol{x}}_{i}}{(n h-i h)^{1 / 2}}\right] \\
& +\frac{0.15 h}{\sqrt{\pi}}\left[\frac{\dot{\boldsymbol{x}}_{n-1}}{h^{1 / 2}}+\frac{2\left(\dot{\boldsymbol{x}}_{n-1}+\dot{\boldsymbol{x}}_{n}\right)}{(0.55 h)^{1 / 2}}+\frac{\dot{\boldsymbol{x}}_{n}}{(0.1 h)^{1 / 2}}\right] \\
& +\frac{0.05 h}{\sqrt{\pi}}\left[\frac{8 \sqrt{2}}{3} \frac{\dot{\boldsymbol{x}}_{n}}{(0.05 h)^{1 / 2}}-\frac{4}{3} \frac{\dot{\boldsymbol{x}}_{n}}{(0.1 h)^{1 / 2}}\right]
\end{aligned}
$$

where $h$ is the timestep, $i$ is the timestep index and $n$ is the index of the most recent computed timestep.

As can be seen in this formulation, the system is storing and reusing the information of the past in order to calculate the current state. Note that the integral in the equation takes all the previous states of the simulation into consideration. At this point, an obvious problem quickly presents itself. Taking into account all the previous states for computing the current state clearly imposes a serious computational barrier. However, a careful analysis of Equation 6 will show that actually only the most recent terms are important to be considered.

Note that the right hand side of Equation 6 is a summation of weighted velocities. The weights in the summation cannot be computed in advance as they are different at each timestep of the simulation. However by analyzing the evolution of these weights (see Figure 6), it is possible to observe that their values decay rapidly as we move away from the current state to the past. Therefore, the most recent states have much more influence on the final result of the equation than the states which are far away in the past and we can safely account only for a few of the most recent states when computing the summation of Equation 6. 

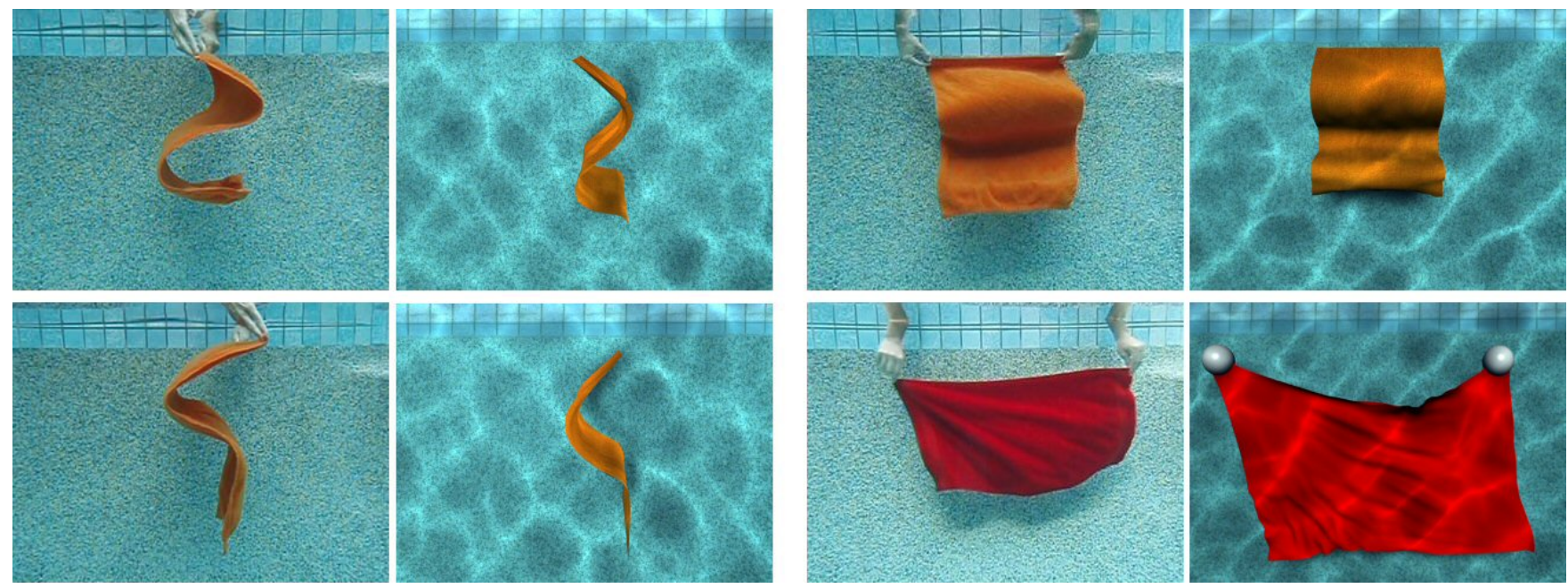

Fig. 5. Comparisons between real clothes and simulated fractional clothes. In each example, a cyclic motion is applied to the top edge of the cloth. The simulations with the orange cloth model produce bumps which are well formed and which propagate in a consistent way, similarly to the ones observed in the real clothes. The red cloth simulation reproduces similar fine details in the interior of the cloth.

To illustrate the evolution of the weights we have computed their values for different simulation settings. The plot in Figure 6 shows the decay of the weights as a function of the timestep index $i$, and for three different values for the timestep $h$. It is possible to observe that the states closer to the most current timestep $(i=n, n-1, n-$ 2) have much greater influence than the states further in the past.

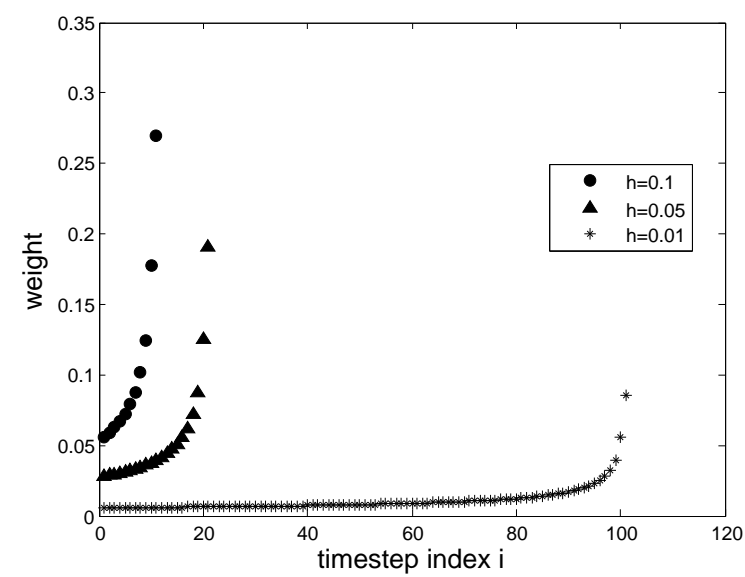

Fig. 6. The plot shows the rapid decay of the weights as we move away from the most recent timestep.

We have also tested experimentally the effect on simulations with different numbers of previous states taken into account. Let $u$ be the number of previous states considered in Equation 6, such that for a given $u$ the summation index $i$ will vary from $n+1-u$ to $n-1$. We indeed noticed that the change on the cloth behavior gets very small for $u>3$. We therefore found $u=3$ to be a good value for the performance/realism tradeoff. While greater values of $u$ will increase the realism of the fluid response effect, the computational cost is also increased.
In Section 5 we present comparative results against real submerged pieces of cloth showing that the chosen $u=3$ achieves sufficiently realistic results.

\subsection{Numerical Integration}

The addition of the fractional derivative terms into the equation of motion of the cloth requires the solution of a Fractional Differential Equation (FDE). Both the explicit and implicit numerical solution techniques available for Ordinary Differential Equations (ODEs) can be extended to FDE's.

Our present work does not focus on developing and comparing different integration methods, and in this paper we have implemented an explicit Euler integration step in order to test and investigate the results achieved by our model. At each timestep the accelerations are computed from the overall force contributions to each particle, and then the system takes one step with:

$$
\begin{aligned}
& \boldsymbol{x}(t+h)=\boldsymbol{x}(t)+h \boldsymbol{v}(t), \\
& \boldsymbol{v}(t+h)=\boldsymbol{v}(t)+h \boldsymbol{a}(t) .
\end{aligned}
$$

The explicit step has been sufficient for the examples simulated in this work and the inclusion of the half-derivative terms does not affect the stability of the integration. Coimbra [2003] suggests an improved explicit method for solving a $2^{\text {nd }}$ order differential equation with a general order damping term by using an adaptation of the Euler method. The approach approximates the first and second derivatives of $\boldsymbol{x}$ using a forward finite-differences method and uses an explicit marching method for the solution of the initial value problem.

Numerical solution techniques for FDEs consisting of an explicit predictor step followed by an implicit corrector step have also been developed [Diethelm et al. 2005; Coimbra C. F. M. 2003]. For stiff equations these methods can be improved by repeatedly iterating the corrector step until a desired convergence is reached. 
In addition to these methods, fully implicit integration schemes such as the approach suggested by Baraff and Witkin [1998] can also be adapted to solve our FDEs. The required partial derivatives of the half-derivative terms can be obtained by computing the partial derivatives of the Riemann-Liouville differential operator (shown in Equation 1).

\section{RESULTS AND DISCUSSION}

Figures 1-3 present several results obtained with our fractional cloth model. In addition, Figures 5-7 present several comparisons demonstrating the capabilities of the model.

Figure 5 illustrates the performed experiments for comparing our model against real pieces of cloth deforming underwater. We tested the behavior of different kinds of fabrics underwater and rectangular cotton towels were the most suitable for the experiment. Several other fabrics just floated because they were not dense enough. Basic patterned movements such as swinging the cloth back and forth, pulling it left and right, etc, were then applied to the immersed pieces of cloth for comparison with our simulated results. These simple motions were selected with the purpose to achieve objective evaluations of the comparisons.

It is possible to notice that when swinging the cloth back and forth the cloth forms deep bumps which slowly and consistently propagate from the source of the applied motion (the top edge) towards the other end of the fabric (the bottom edge). The bumps consistently last until the bottom edge of the cloth is reached and this behavior is a good justification of how fractional derivatives with their history-laden properties are suitable for simulating an underwater cloth. Fractional clothes are able to realistically simulate these observed effects.

Figure 7 compares our fractional model with three regular cloth simulation models based on damped springs subjected to different drag forces. Note that all the clothes are under water buoyancy and the first three models do not include fractional terms. The first cloth model is affected by both viscous drag and normal drag forces. The second model is only affected by viscous drag forces. In the third model we only used normal drag forces. The employed normal drag force worked best in its linear form: $-k_{d}(\dot{\boldsymbol{x}} . \hat{\boldsymbol{n}}) \hat{\boldsymbol{n}}$. In all cases we fine-tuned the drag coefficients in order to get as close as possible to the observed underwater behavior. These three regular models represent our best attempts to achieve underwater effects by tuning as most as possible the parameters of regular cloth deformation systems. As can be observed in Figure 7, although regular models are able to form some of the observed bumps, they are not always well shaped and several times they are overly long, covering the whole extent of the fabric and giving the cloth a bended look. The longlasting shape and the consistent propagations cannot be correctly simulated with regular models.

In the right-most column of Figure 7 we show results obtained by our fractional cloth model. The parameters are the same as the ones used in the regular models, but now the internal damped springs are replaced by the viscoelastic spring elements and the history drag force $\boldsymbol{F}_{h d}$ is added. We can therefore evaluate the new effects produced by the half-derivative terms. The comparisons in Figure 7 show that fractional clothes are able to best reproduce the behavior of the real immersed clothes. The simulations formed well-shaped and deep bumps which were locally formed near the motion source (the top edge) and did not cover the whole extent of the cloth, similarly to the behavior observed in the real clothes.
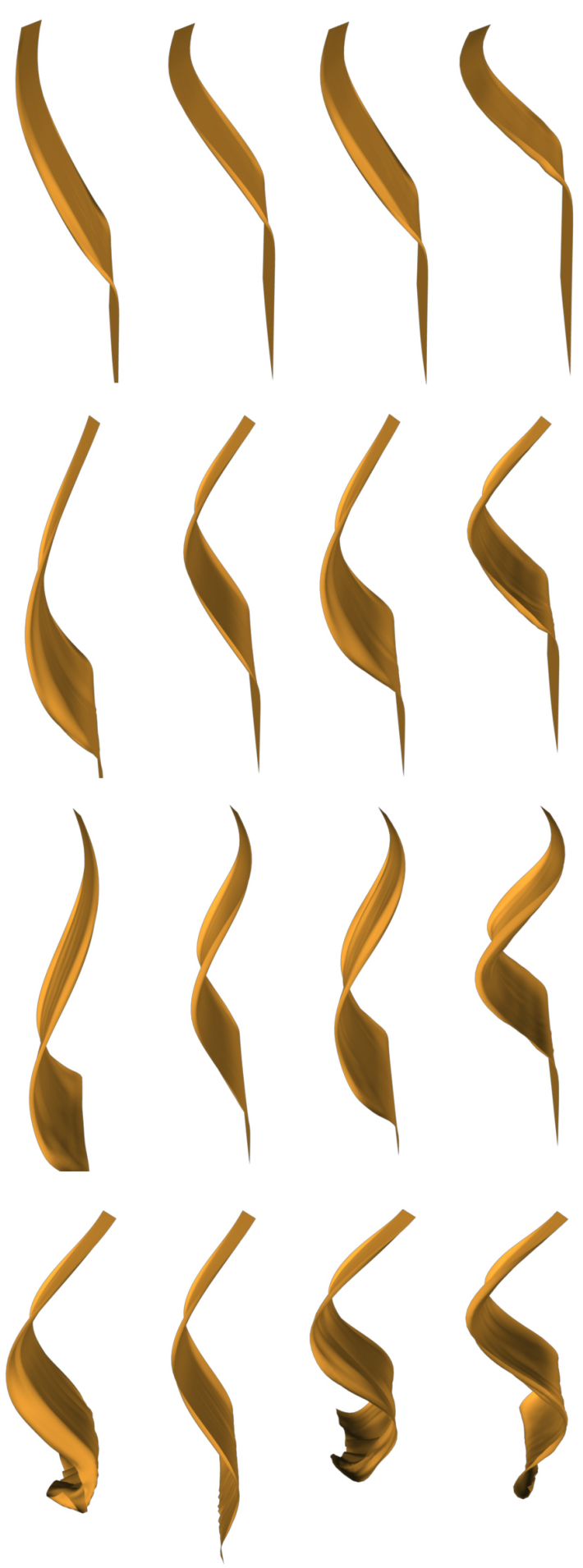

Fig. 7. Comparisons between regular cloth deformation models using various drag models (left three columns) and fractional clothes (right-most column). It is possible to notice that fractional clothes always produce more realistic bump formations. For instance in the bottom-most comparison only the fractional cloth model is able to produce three well formed bumps. 
While the performed comparisons (Figures 5 and 7) provide objective evaluations of our method in respect to global behaviors, the additional animations presented in Figures 1, 2 and 3 show several realistic local deformation effects obtained.

We have also compared the behavior of our fractional cloth model against a regular model in a situation with more energetic motions. The simulated scene consists of a cloth model colliding and interacting with a moving solid sphere, as shown in the left two images of Figure 3. The same simulation was performed with both our fractional model and our best regular cloth model, which is the one using only normal drag forces. The obtained animations show that our fractional model can clearly generate waves on the cloth which propagate more consistently and which last longer, in comparison with the regular model. These animations are available in the videos accompanying this paper.

Our simulations were conducted on a $2.4 \mathrm{GHz}$ Intel Core 2 Duo desktop computer. Cloth meshes of both 2501 particles in a $61 \times 41$ mesh grid and 651 particles in a $31 \times 21$ mesh grid were used in the swinging simulations in order to observe the resulting effects of fractional terms in models with different resolutions. These simulations were performed with $u=3$, i.e., using 3 states in the past for the computation of Equation 6. In order to handle collisions, these simulations also include a standard collision avoidance repulsive force opposing the direction of potential collisions. The implementation of the cloth with $31 \times 21$ mesh grid achieved the average computation time of about 9 frames per second for each cloth piece. The same simulations, when performed with the regular cloth models (without the fractional terms), could be computed in the order of 2 to 3 times faster, however without achieving the underwater behaviors. In contrast, reported times taken by complete fluid simulation systems commonly run in the order of a few minutes to several hours per frame of simulation in high performance machines [Robinson-Mosher et al. 2008].

One of the caveats of our approach is that the large scale interactions of the flow with the cloth are not modeled. The same is true for the flow disturbances caused by the cloth from other regions in the flow. The model does however include the implicit effect of the presence of the cloth in the flow in the vicinity of each node of the calculation, and with this effect it is able to produce realistic results at a small fraction of the computational resources needed to model the two-way coupling between the flow and the cloth.

\section{CONCLUSIONS}

We have introduced a new methodology for modeling immersed deformable models which is based on the use of Fractional Calculus and we have demonstrated that our fractional cloth model is able to achieve realistic underwater behaviors. The history terms introduced by the half-derivatives prove to be very effective in achieving the expected viscoelastic behaviors of the immersed cloth.

The fact that realistic behaviors can be achieved without modeling or simulating the body of water is remarkable and the proposed approach will prove itself useful to a number of applications employing deformable objects. For instance, the presented methodology can be integrated into the motion equations of any particle-based structures such as the ones used in hair simulation and in several volumetric models common in surgical simulations. We believe that fractional derivatives coupled with physically based models have the potential to impact several applications in computer graphics.
Acknowledgments We would like to thank Robert Backman for the valuable help with the character modeling and with the preparation of the accompanying videos.

\section{REFERENCES}

BARAFF, D. AND WitKIn, A. 1997. Physically based modeling: Principles and practice. In ACM SIGGRAPH '97 Course Notes.

BARAFF, D. AND WitKIn, A. 1998. Large steps in cloth simulation. In Proc. of SIGGRAPH'98. New York, NY, USA, 43-54.

BASSET, A. B. 1888. On the motion of a sphere in a viscous liquid. Transactions of the Royal Philosophy Society of London 179, 43-63.

BoussinesQ, J. 1885. Sur la résistance qu'oppose un liquide indéfini en repos, sans pesanteur, au mouvement varié d'une sphère solide qu'il mouille sur toute sa surface, quand les vitesses restent bien continues et assez faibles pour que leurs carrés et produits soient négligeables. $C$. $R$. Acad. Sci., 935-937.

Breen, D., House, D., And Wozny, M. 1992. Predicting the drape of woven cloth using interacting particles. In Proc. of SIGGRAPH'94. ACM, New York, NY, USA, 365-372.

Carignan, M., Yang, Y., Magenenat-Thalmann, N., And ThalMANN, D. 1992. Dressing animated synthetic actors with complex deformable clothes. In Proc. of SIGGRAPH'92. ACM, New York, NY, USA, 99-104.

Choi, K. And Ko, H. 2002. Stable but responsive cloth. In Proc. of SIGGRAPH'02. ACM, New York, NY, USA, 604-611.

Coimbra C. F. M. 2003. Mechanics with variable order operators. Annalen der Physik 12, 692-703.

Coimbra C. F. M. AND Kobayashi M. H. 2002. On the viscous motion of a small particle in a rotating cylinder. Journal of Fluid Mechanics 469, 257-286.

Coimbra C. F. M., L'Esperance, D., Lambert, A., Trolinger J. D., AND RANGel R. H. 2004. An experimental study on the history effects in high-frequency Stokes flows. Journal of Fluid Mechanics 504, 353-363.

Coimbra C. F. M. And Rangel R. H. 1998. General solution of the particle equation of motion in unsteady Stokes flows. Journal of Fluid Mechanics 370, 53-72.

Desbrun, M., SchröDER, P., AND BARR, A. 1999. Interactive animation of structured deformable objects. In Proc. of Graphics interface. San Francisco, CA, USA, 1-8.

Diethelm, K., Ford, N., Freed, A., And LuchKo, Y. 2005. Algorithms for the fractional calculus: A selection of numerical methods. Computer Methods in Applied Mechanics and Engineering 194, 743773.

Eberhardt, B., Weber, A., AND Strasser, W. 1996. A fast, flexible, particle-system model for cloth draping. In IEEE Computer Graphics and Applications'96. IEEE, 52-59.

Hauth, M., Etzmuss, O., And Strasser, W. 2003. Analysis of numerical methods for the simulation of deformable models. The Visual Computer 19, 7-8, 581-600.

Hilfer, R. 2000. Applications of Fractional Calculus in Physics. World Scientific, River Edge, NJ.

House, D. H. AND BreEn, D. E., Eds. 2000. Cloth modeling and animation. A. K. Peters, Ltd., Natick, MA, USA.

HU, Y. 2005. Integral transformations and anticipative calculus for fractional brownian motions. In Memoirs of the American Mathematical Society.

Kilbas, A., Srivastava, H., And Trujillo, J. 2006. Theory and Applications of Fractional Differential Equations. Amsterdam, The Netherlands. 
LARSON, R. G. 1998. The Structure and Rheology of Complex Fluids. Oxford University Press, USA.

L'Esperance, D., Coimbra C. F. M., Trolinger J. D., AND RAngel R. H. 2005. Experimental verification of fractional history effects on the viscous dynamics of small spherical particles. Experiments in Fluids 38, $112-116$.

LING, L. 2000. Aerodynamic effects. In Cloth modeling and animation, D. H. House and D. E. Breen, Eds. A. K. Peters, Ltd., Natick, MA, USA, 175,195 .

Ling, L., Damodaran, M., And Gay, R. K. L. 1996. Aerodynamic force models for animating cloth motion in air flow. The Visual Computer 12, 2, 84-104.

Miller, K. AND Ross, B. 1993. An Introduction to the Fractional Calculus and Fractional Differential Equations. John Wiley and Sons, New York, NY.

Müller, M., Schirm, S., Teschner, M., Heidelberger, B., AND GROss, M. 2004. Interaction of fluids with deformable solids: Research articles. Computer Animation and Virtual Worlds 15, 3-4, 159-171.

Oldham, K. AND Spanier, J. 1974. The Fractional Calculus. Academic Press, New York, NY.

Podlubny, I. 1999. Fractional Differential Equations. Academic Press, San Diego, CA.

Provot, X. 1995. Deformation constraints in a mass-spring model to describe rigid cloth behavior. In Proc. of Graphics Interface '95. New York, NY, USA, 147-155.

Robinson-Mosher, A., Shinar, T., Gretarsson, J., Su, J., And FEDKIw, R. 2008. Two-way coupling of fluids to rigid and deformable solids and shells. ACM Transactions on Graphics (Proc. of SIGGRAPH'08) 27, 3, 1-9.

Selle, A., Su, J., Irving, G., And Fedkiw, R. 2009. Robust highresolution cloth using parallelism, history-based collisions, and accurate friction. Transactions on Visualization and Computer Graphics 15, 2.

STOKES, G. G. 1845. On the theories of internal friction of fluids in motion. Transactions of the Cambridge Philosophy Society 8, 287-305.

Terzopoulos, D. And Fleischer, K. 1988. Deformable models. The Visual Computer 4, 6, 306-331.

Terzopoulos, D., Platt, J., Barr, A., and Fleischer, K. 1987. Elastically deformable models. In Proc. of SIGGRAPH '87. ACM, New York, NY, USA, 205-214.

Tu, X. And Terzopoulos, D. 1994. Artificial fishes: physics, locomotion, perception, behavior. In Proc. of SIGGRAPH '94. ACM Press, New York, NY, USA, 43-50.

Volino, P., Courchesne, M., And Thalmann, N. M. 1995. Versatile and efficient techniques for simulating cloth and other deformable objects. In Proc. of SIGGRAPH'95. ACM, New York, NY, USA, 137-144. 